\title{
Les élections locales 2013 en Côte d'Ivoire : des malentendus subsistent sur l'exercice de la démocratie
}

\section{Christian Bouquet et Irène Kassi-Djodjo}

\section{(2) OpenEdition}

1 Journals

\section{Édition électronique}

URL : http://journals.openedition.org/echogeo/13697

DOI : 10.4000/echogeo.13697

ISSN : 1963-1197

Éditeur

Pôle de recherche pour l'organisation et la diffusion de l'information géographique (CNRS UMR 8586)

Référence électronique

Christian Bouquet et Irène Kassi-Djodjo, « Les élections locales 2013 en Côte d'Ivoire : des malentendus subsistent sur l'exercice de la démocratie », EchoGéo [En ligne], Sur le Vif, mis en ligne le 13 janvier 2014, consulté le 02 mai 2019. URL : http://journals.openedition.org/echogeo/13697 ; DOI 10.4000/echogeo.13697

Ce document a été généré automatiquement le 2 mai 2019.

\section{c) (7) $९$}

EchoGéo est mis à disposition selon les termes de la licence Creative Commons Attribution - Pas d'Utilisation Commerciale - Pas de Modification 4.0 International 


\title{
Les élections locales 2013 en Côte d'Ivoire : des malentendus subsistent sur l'exercice de la démocratie
}

\author{
Christian Bouquet et Irène Kassi-Djodjo
}

1 Poursuivant son retour à un fonctionnement démocratique normal, la Côte d'Ivoire a organisé en avril 2013 les dernières élections prévues pendant la mandature d'Alassane Ouattara, c'est-à-dire les municipales et les régionales. Après avoir respecté le calendrier du scrutin législatif (décembre 2011) qui devait suivre l'élection présidentielle, les autorités ont invité les électeurs à procéder à la désignation des conseillers municipaux et des conseillers régionaux le 21 avril 2013. Si cette nouvelle consultation s'est déroulée sans incidents majeurs, la plupart des problèmes qui s'étaient posés au lendemain de la crise postélectorale (décembre 2010 - avril 2011) demeurent intacts (Bouquet, KassiDjodjo, 2012).

2 Outre le fait que l'usage de la démocratie n'est toujours pas parfaitement maîtrisé, on a pu observer - le cas de la Côte d'Ivoire n'étant d'ailleurs pas isolé - que le transfert des modèles du Nord dans les pays du Sud rencontre toujours des difficultés. Ainsi la décentralisation, notamment dans sa déclinaison électorale, a-t-elle du mal à devenir une réalité tangible et, surtout, à convaincre de son efficacité.

\section{Un territoire redécoupé, et des centaines de milliers d'électeurs encore oubliés}

3 C'est ainsi qu'après avoir redessiné les circonscriptions électorales des députés, les autorités ivoiriennes ont entrepris de détricoter le maillage municipal que le régime de Laurent Gbagbo avait mis en place entre 2001 et 2010. Il est vrai que ce dernier avait multiplié les créations, faisant passer le nombre de communes de 191 à 1 323. En fait, les communes ainsi créées n'étaient pas fonctionnelles et n'existaient que de nom. Seules des 
élections locales auraient $\mathrm{pu}$ en faire de véritables municipalités. Certes, cela correspondait à une demande des populations qui pensaient pouvoir ainsi amorcer le développement de leurs localités. Mais, davantage qu'un soutien au processus de déconcentration-décentralisation, il s'agissait surtout, pour le pouvoir de l'époque, de créer des emplois d'agents municipaux (à la charge du budget de l'État) et d'ouvrir des niches et prébendes pour les amis politiques.

C'est au moment du redécoupage que l'idée de supprimer 1126 communes a germé dans l'esprit des nouvelles élites au pouvoir et, en avril 2012, on est revenu à 197 collectivités municipales, soit pratiquement les mêmes que celles qui avaient donné lieu à des élections en 2001. En juillet 2012, une dernière main a été portée au redécoupage régional entrepris en 2011 (Bouquet et Kassi-Djodjo, 2012) avec la scission de la région du Nzi et la création de celle du Moronou, portant ainsi à 31 le nombre de régions qui allaient être soumises à des élections pour désigner les conseillers régionaux.

5 L'architecture administrative de la Côte d'Ivoire a donc été arrêtée comme suit: 14 districts - dont deux autonomes, ceux d'Abidjan et de Yamoussoukro - chapeautent 31 régions, divisées en 107 départements et 510 sous-préfectures. Les districts sont dirigés par des gouverneurs, c'est-à-dire des superpréfets nommés par le pouvoir, sans représentation démocratique. Parallèlement, les conseils généraux qui cogéraient les départements avec les préfets disparaissent. En «allégeant» ainsi la démocratie territoriale, l'État ivoirien réalise de grosses économies et peut éviter de passer par des rouages politiques susceptibles de retarder ou de bloquer les projets de développement.

On comprend alors que les élections municipales et régionales de 2013 se sont inscrites dans des enjeux limités, sachant par ailleurs que les transferts de compétences - et surtout de moyens - sont loin d'avoir été intégralement réalisés (voir infra).

7 Par ailleurs, la logique spatiale du redécoupage reposait sur la volonté de s'adapter aux évolutions démographiques ayant marqué la dernière décennie, du moins telles qu'on les supposait car aucune enquête n'avait été faite depuis le recensement de 1998. Pour autant, les autorités ne sont pas allées plus loin que le tracé de nouvelles circonscriptions, et c'est ainsi que les listes électorales, reconstituées a minima pour l'élection présidentielle de 2010, n'ont pas été touchées pour les législatives de 2011, et pas davantage pour les municipales et les régionales de 2013. On se souvient que les opérations d'enrôlement - qui avaient duré trois ans et suscité plusieurs polémiques avaient abouti à l'identification d'environ 5700000 électeurs autorisés à voter en octobre 2010, soit à peine 200000 de plus que pour l'élection présidentielle d'octobre 2000. Cela équivalait à une croissance démographique de $0,3 \%$ par an, alors que des sondages laissent entendre que cette croissance continue de dépasser $3 \%$ par an... À l'évidence, il manque au moins 2500000 électeurs sur les listes, et probablement davantage, soit 35 à $40 \%$ des Ivoiriens majeurs qui se trouvent toujours écartés des urnes.

Pendant la campagne électorale pour les élections locales, l'opinion publique n'a pas semblé sensible à cet accroc à la démocratie, d'abord parce que la rumeur de l'imminence d'un nouveau recensement ${ }^{1}$ a circulé avec insistance, et ensuite parce que la question de la nationalité suscite encore des réactions politico-épidermiques qui poussent les autorités à reporter le débat le plus tard possible, de peur de voir le pays de nouveau s'enflammer'.

9 En avril 2013, 3794774 électeurs ont donc été conviés à participer aux élections municipales dans 197 communes, contre - douze ans plus tôt - 3188144 pour 
191 communes (les mêmes, moins six nouvelles, ce qui pourra favoriser les études diachroniques). Cela revient à admettre que la population de ces communes, qui sont des aires urbaines de moyenne et de grande importance, n'a crû que de 1,4 \% par an pendant toute cette période. On peut imaginer la frustration des Ivoiriens privés de vote depuis 2002 , et celle des "nouveaux majeurs ", mais force est de constater que la campagne électorale n'en a pas été affectée.

\section{Des partis politiques au fonctionnement anachronique} manière plutôt apaisée. Il y avait d'ailleurs si peu d'engouement pour ces consultations que la date initialement prévue pour le dépôt des candidatures (23 février 2013) avait dû être reportée, car la CEI (Commission électorale indépendante) n'avait enregistré que 49 dossiers pour les 197 communes, et aucun pour les 31 régions...

11 En fait, le principal sujet de préoccupation était - comme pour les législatives l' « inclusivité », concept relativement nouveau dans la géopolitique africaine et qui signifie que toutes les composantes politiques doivent participer.

Rappelons les grands traits du paysage politique ivoirien (Bouquet, 2011), tel qu'il se présentait à son moment le plus clair, c'est-à-dire lors du premier tour de l'élection présidentielle (31 octobre 2010) : le FPI $^{3}$ de Laurent Gbagbo recueillait $38 \%$ des voix, contre $32 \%$ pour le RDR d'Alassane Ouattara, et $25 \%$ pour le PDCI d'Henri Konan Bédié. Au second tour (28 novembre 2010), l'alliance du RDR et du PDCI, rassemblée depuis 2005 au sein du RHDP (Rassemblement des Houphouëtistes pour la Démocratie et le Progrès), l'avait emporté avec $54 \%$ des suffrages pour Alassane Ouattara contre $46 \%$ à Laurent Gbagbo. Ce dernier n'ayant pas accepté le résultat, il s'en était suivi une longue crise, presque une guerre civile, à l'issue de laquelle Alassane Ouattara avait pu s'installer au pouvoir (avril 2011), tandis que Laurent Gbagbo, après plusieurs mois de résidence surveillée, avait été transféré à La Haye à la demande de la CPI (Cour pénale internationale) pour répondre de plusieurs chefs d'accusation passibles du Tribunal pénal international (novembre 2011).

De même que les élections législatives de décembre 2011 avaient été marquées par le refus du FPI de revenir dans le jeu politique «tant que Laurent Gbagbo et tous les cadres du parti n'auraient pas été libérés", les élections municipales et régionales ont été boycottées, puisque la situation n'avait pas bougé dans ce domaine. Le parti vaincu a continué à se crisper sur une perception étroite de la "réconciliation nationale ", allant jusqu'à exiger non seulement le renouvellement complet de la $\mathrm{CEI}^{4}$ - ce qui n'était pas illogique - mais aussi une amnistie générale voire, pour certains d'entre eux, l'annulation du second tour de l'élection présidentielle de $2010 . .$.

Malgré plusieurs mains tendues de la part du pouvoir en place, le FPI a donc maintenu sa position de non-participation, suivi d'ailleurs par le petit parti LIDER (Liberté et Démocratie pour la République) de Mamadou Koulibaly, ex-président (FPI) de l'Assemblée nationale, qui semblait pourtant prêt à jouer le jeu des urnes puisqu'il avait été candidat (malheureux) à la députation. Dans ce dernier cas, il est probable que le scrutin de liste caractérisant les élections locales (municipales et régionales) a compliqué la tâche d'un groupe comptant de faibles effectifs. 
15 Mais, au-delà du cas du FPI, le fonctionnement des appareils politiques ivoiriens en général n'a pas évolué avec le temps, et n'est donc plus tout à fait en phase avec la réalité d'un processus démocratique bien compris. C'est ainsi que, faisant fi des injonctions de l'ancien parti au pouvoir, plusieurs dizaines de candidats FPI ont fait campagne sous l'étiquette des « indépendants ». De même, de nombreux candidats RDR ou PDCI n'ayant pas obtenu l'investiture de leurs bureaux politiques respectifs sont également allés aux élections en tant qu' « indépendants ».

Tableau 1 - Candidatures (listes) aux élections locales

\begin{tabular}{|l|l|l|l|l|l|}
\hline & Indépendants & RDR & PDCI & UDPCI & RHDP \\
\hline Régionales (31 régions) & 40 & 19 & 12 & & 9 \\
\hline Municipales (197 communes) & 344 & 166 & 122 & 13 & 5 \\
\hline
\end{tabular}

16 Les grandes batailles internes aux partis pour les investitures ont souligné l'attachement à une forme de gérontocratie qui perdure et paralyse tout mouvement de renouvellement des élites. Pendant que certaines voix du FPI prétendaient qu'ils ne pouvaient présenter personne aux élections locales " parce que leurs cadres étaient tous en prison », le RDR et le PDCI connaissaient des grincements internes liés au fait qu'ils promouvaient d'abord tous leurs caciques. C'est ainsi que la plupart des ministres ont brigué les postes de maires ou de présidents des conseils régionaux, écornant ainsi leur image dans l'esprit de leurs électeurs : pouvait-on, dans cette nouvelle démocratie, être à la fois ministre et président de région?

17 Outre le fait que la dynamique d'alliance qui avait permis au RHDP de porter Alassane Ouattara au pouvoir semblait oubliée, surtout pour les municipales ( 5 candidatures d'alliance contre 301 candidatures relevant individuellement des partis membres du RHDP), les appétits personnels et les calculs politiciens ont donc poussé des centaines de candidats dans les rangs hétéroclites des "indépendants", rendant ainsi difficile la lecture du paysage politique. Car derrière les 40 têtes de listes pour les régionales et les 344 têtes de listes pour les municipales, des milliers d'autres « indépendants » ont brigué les postes de conseillers régionaux ou de conseillers municipaux.

\section{Le RDR sort vainqueur, mais a-t-il vraiment gagné ?}

Globalement, ces élections locales se sont déroulées sans incidents notables. Certes, il y a eu des contestations, et 186 recours ont été enregistrés, mais quatre seulement (communes de Anyama, Doropo et Bettié, et région de Tchologo) ont été retenus, sur la base d'éléments avérés de violences ou de fraude. Des élections partielles ont eu lieu le 21 juillet 2013, et elles ont confirmé les premiers résultats.

19 On a d'ailleurs remarqué - et cela mérite d'être souligné - que les mauvais perdants appartenaient surtout à la majorité présidentielle. À Séguéla, le secrétaire général par intérim du RDR, Amadou Soumahoro, a été battu par un indépendant (en réalité un candidat RDR qui n'avait pas obtenu l'investiture de son parti). De même, les très médiatiques ministres Cissé Bacongo (rénovateur de l'université de Cocody) et Anne 
Oulotto (surnommée «tantie bulldozer» en raison de son efficacité dans les " déguerpissements ») ont trébuché et ont protesté de manière disproportionnée. Ici et là, quelques membres de la CEI (Commission électorale indépendante) sont redevenus des militants passionnés du RDR ou du PDCI, et ont transgressé leur code moral.

Il reste donc du chemin à faire avant d'atteindre la démocratie bien comprise et apaisée vers laquelle devrait tendre la Côte d'Ivoire, pourtant échaudée par douze années de dérapages divers. Force est cependant de constater que l'exécutif n'a pas interféré pour « rectifier » des résultats défavorables à certains leaders du RDR.

21 Pour évaluer la légitimité des deux consultations, on a évidemment scruté la participation, notamment parce que le FPI, qui les boycottait, entendait montrer implicitement sa force en s'attribuant les abstentionnistes. Son calcul était osé, car la participation a été de $44,37 \%$ aux régionales (contre $28,01 \%$ aux départementales de 2002, seule élection comparable), et de $36,56 \%$ aux municipales (contre $39,35 \%$ aux municipales de 2001). Cette dernière comparaison, prise au pied de la lettre, indique qu'il y a eu 2,79 \% d'abstentionnistes en plus en 2013. Est-ce le poids du FPI?

22 Lorsque les premiers résultats sont tombés, une partie de la presse a titré : «Victoire des indépendants ». Effectivement, ceux-ci arrivaient en tête pour les municipales, aussi bien par le nombre de communes remportées (71) que par le total des voix (494 198) et le pourcentage des suffrages $(36,92 \%)$. De même, ils affichaient un score très élevé aux régionales puisqu'ils talonnaient le RHDP en voix (455 669 contre 460678 ) et donc en pourcentage $(28,31 \%$ contre $28,63 \%)$.

23 Mais quelques jours plus tard certains de ces « indépendants » se sont démasqués et ont rallié le RDR (dans 27 communes et une région). La carte des élections municipales reflète donc la très forte poussée du RDR, parti au pouvoir depuis avril 2011. 


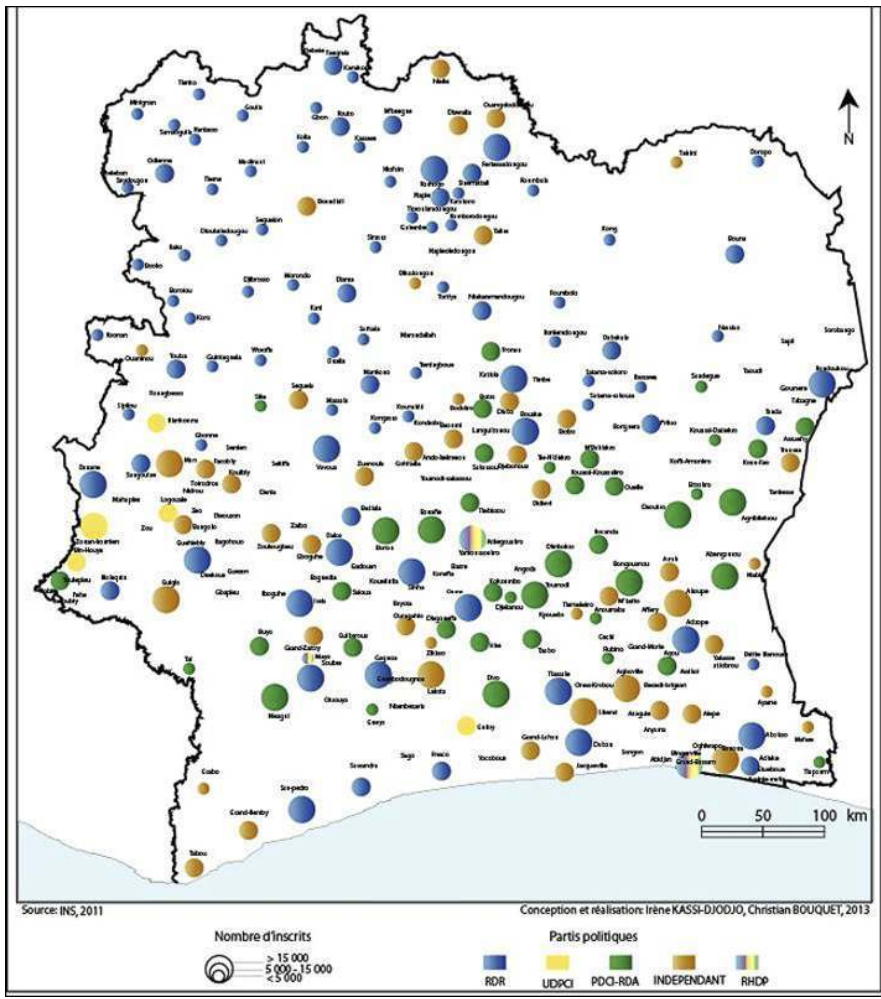

Source : INS, 2011 ; conception et réalisation : I. Kassi Djodjo, C. Bouquet, 2013

Tableau 2 - Comparaison des résultats 2001-2013 aux élections municipales (nombre de communes et voix)

\begin{tabular}{|l|l|l|}
\hline & Municipales (25/03/2001) & Municipales (21/04/2013) \\
\hline FPI & $33(278774)$ & Boycott \\
\hline PDCI & $60(298206)$ & $48(398210)$ \\
\hline RDR & $63(301179)$ & $\mathbf{9 6}(510969)$ \\
\hline Indépendants & $35(218774)$ & $44(418726)$ \\
\hline UDPCI & & $5(30441)$ \\
\hline RHDP & & $4(49183)$ \\
\hline & 191 communes & 197 communes \\
\hline
\end{tabular}

Mais qu'en a-t-il été des 33 communes tenues depuis 2001 par le FPI, puisqu'il a boycotté ? Sept d'entre elles ont été implicitement « conservées », puisque les « indépendants » qui les ont remportées étaient membres ou sympathisants du FPI. Treize autres sont allées au PDCI (dont la commune abidjanaise de Cocody) et neuf au RDR (dont la commune abidjanaise de Yopougon, fief urbain de Gbagbo). Dans ces deux cas, la participation n'a 
été respectivement que de 15,2 et 16,0\%. Ici, le FPI peut légitimement mesurer par défaut son poids électoral.

Illustration 2 - Résultats des élections municipales de 2013 dans le Grand-Abidjan

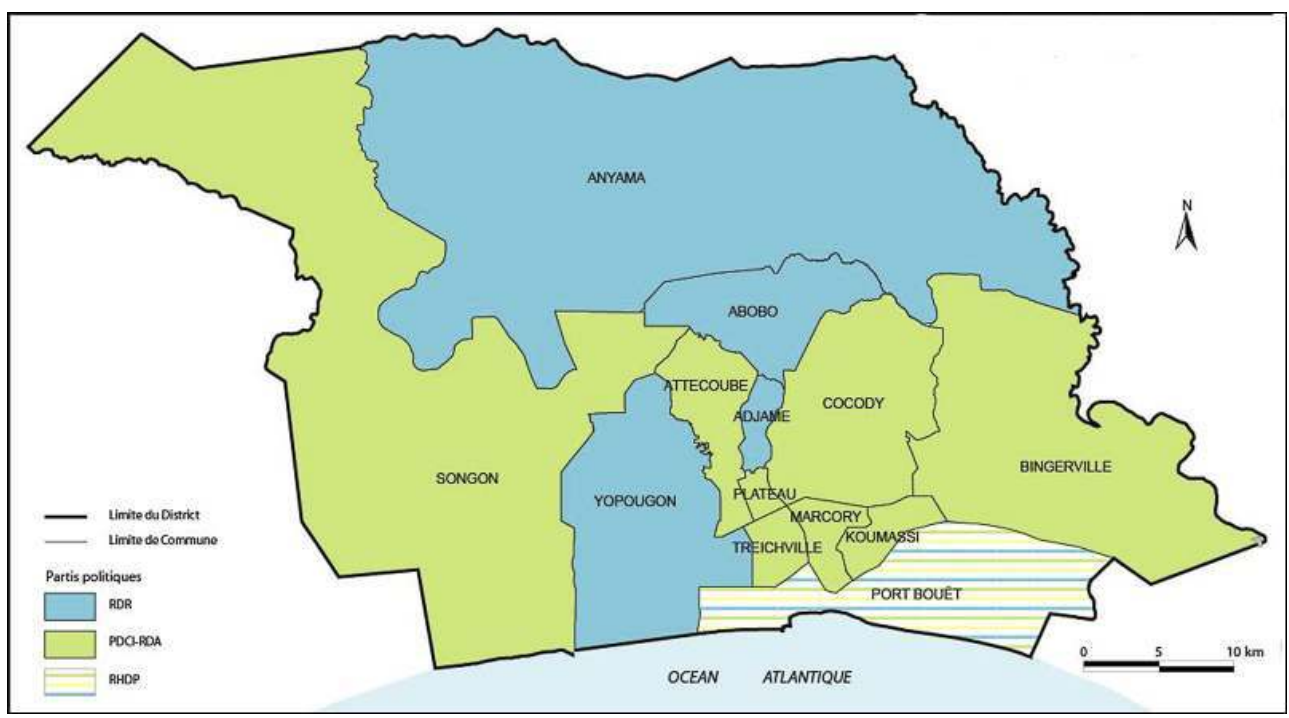

S'agissant d'une carte de géographie politique, les auteurs ont volontairement occulté le tracé des lagunes.

Source : CCT, 2000 ; résultat et conception : C. Bouquet, I. Kassi-Djodjo, 2013.

Si l'on s'en tient au nombre de communes remportées, le tableau 2 laisse entendre que le RDR a largement dominé les élections municipales, tandis que le PDCI, pourtant en progression dans les suffrages, a perdu 12 communes. Sur les dix communes d'Abidjan, on croit déceler une érosion de l'électorat RDR depuis les législatives de décembre $2011 \mathrm{si}$ l'on se contente d'additionner les voix obtenues par les candidats RDR vainqueurs. Mais, en approfondissant les recherches, on découvre que sur 38 candidatures dites « indépendantes », 15 étaient des PDCI dissidents et 14 des RDR dissidents.

Un sondage effectué dans les 22 communes de trois régions du Nord (Tchologo, Bagoué et Poro) indique également que 13 des 17 candidats «indépendants » battus étaient en réalité des dissidents RDR. Cette situation montre, d'une part, à quel point la catégorie des indépendants est difficile à décrypter et, d'autre part, que les grands partis - et notamment le RDR - rencontrent des difficultés à faire respecter leurs arbitrages lors des investitures. Dans certaines communes urbaines, on a compté jusqu'à quatre candidats officiellement indépendants mais en réalité dissidents RDR engagés jusqu'au bout contre le candidat officiel de leur propre parti.

Tableau 3 - Évolutions en voix des principaux partis dans les 10 communes d'Abidjan (2000/2013)

\begin{tabular}{|c|c|c|c|c|c|}
\hline & $\begin{array}{l}\text { Législatives } \\
2000\end{array}$ & $\begin{array}{l}\text { Municipales } \\
2001\end{array}$ & $\begin{array}{l}\text { Présidentielle } \quad 1^{\text {er }} \\
\text { tour } 2010\end{array}$ & $\begin{array}{l}\text { Législatives } \\
2011\end{array}$ & $\begin{array}{l}\text { Municipales } \\
2013\end{array}$ \\
\hline FPI & 199183 & 135436 & 597245 & boycott & boycott \\
\hline RDR & boycott & 117254 & 446528 & 220683 & $147213(193223$ \\
\hline
\end{tabular}




\begin{tabular}{|l|l|l|l|l|l|}
\hline PDCI & 131691 & 93053 & 269495 & 96025 & $\begin{array}{l}136417 \text { (164 035 } \\
\text { (25 }\end{array}$ \\
\hline
\end{tabular}

En italique, le nombre de voix « corrigé » par la prise en compte des « indépendants dissidents »)

L'analyse des résultats des élections régionales est plus délicate à conduire, dans la mesure, d'une part, où les districts autonomes d'Abidjan et de Yamoussoukro en étaient exclus et, d'autre part, où les listes d'union RHDP (RDR + PDCI + UDPCI) étaient beaucoup plus présentes qu'aux municipales. Par ailleurs, étant les premières du genre, ces élections régionales ne permettent guère les comparaisons.

\section{Illustration 3 - Résultats des élections régionales de 2013 en Côte d'Ivoire}

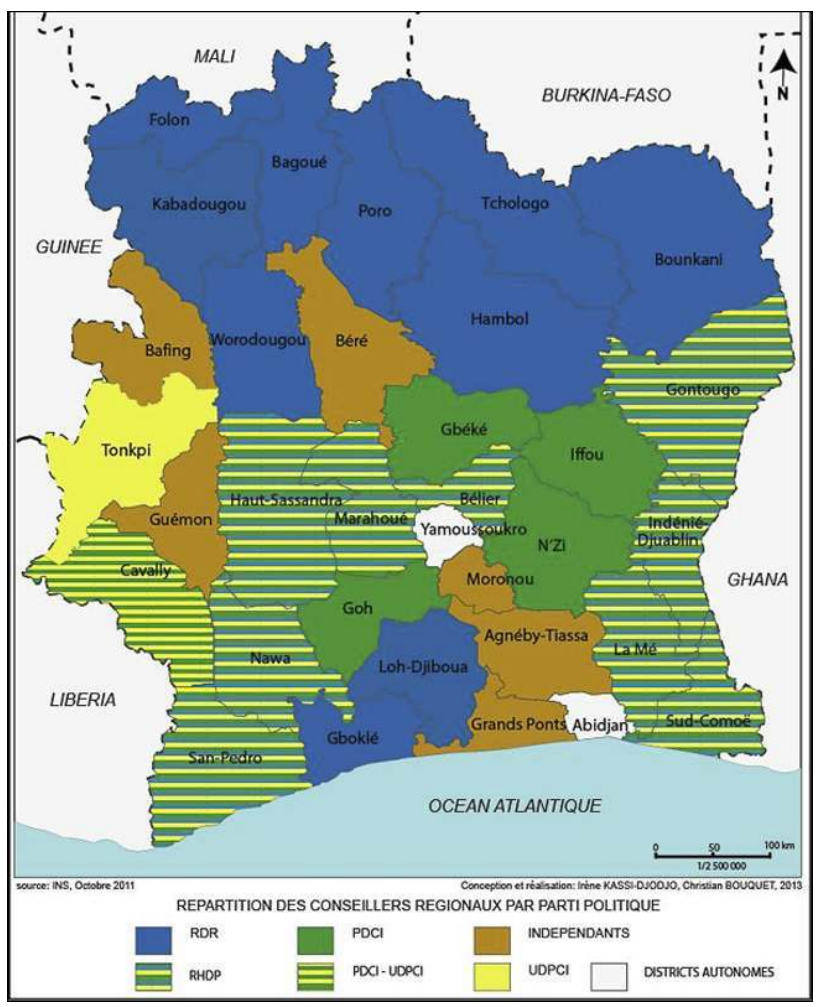

Source : INS, octobre 2011 ; conception et réalisation : I. Kassi-Djodjo, C. Bouquet, 2013.

Pour autant, la carte est plus claire que celle des municipales. Le RDR conserve ses fiefs septentrionaux, tandis que le PDCI semble se contenter de la portion congrue (4 régions pour $14 \%$ des suffrages exprimés). Mais ces résultats bruts sont masqués par la vague RHDP qui arrive en tête en nombre de voix. L'actuel chef de l'État peut y voir un signe positif pour la stratégie de l'union, sans laquelle il n'est pas sûr de pouvoir être réélu. Néanmoins, en cas de retour du FPI dans le jeu politique, les cartes pourraient être rebattues.

Tableau 4 - Résultats des élections régionales en 2013

\begin{tabular}{|l|l|l|l|}
\hline & régions & voix & $\%$ des suffrages \\
\hline Indépendants & 5 & 441907 & $27,46 \%$ \\
\hline
\end{tabular}




\begin{tabular}{|l|l|l|l|}
\hline RDR & 11 & 351956 & $21,87 \%$ \\
\hline PDCI & 4 & 225091 & $13,99 \%$ \\
\hline RHDP & 9 & 460678 & $28,63 \%$ \\
\hline UDPCI & 1 & 76266 & $4,74 \%$ \\
\hline PDCI-UDPCI & 1 & 14517 & $0,90 \%$ \\
\hline & 31 & & \\
\hline
\end{tabular}

Pas davantage que les municipales, les élections régionales n'ont facilité la promotion de jeunes cadres politiques issus des grands partis en présence. Au contraire, ce sont pour la plupart des ministres ou d'anciens ministres qui occuperont le fauteuil de président de région. Certes les pays du Nord - et singulièrement la France - n'ont guère de leçon à donner dans ce domaine, mais la montée en puissance des jeunes générations est quantitativement plus forte dans les pays du Sud, et leur impatience souvent plus grande.

Il est vrai que les conseils municipaux ou régionaux sont encore trop souvent des sinécures, et non pas (encore) des lieux d'apprentissage de la gestion décentralisée des services publics, car les périmètres d'intervention demeurent étroits.

\section{Les compétences (et les moyens) réellement transférés aux collectivités territoriales sont encore limités}

Dernier en date des transferts de modèles, la « décentralisation » a souvent été présentée ( " vendue »?), notamment par les institutions internationales, comme la formule miracle pour rendre les collectivités territoriales plus autonomes et, plus globalement, pour remplir les critères de la bonne gouvernance. En Afrique, les pouvoirs en place y ont trouvé un intérêt supplémentaire: l'opportunité de "caser» dans les provinces les personnels politiques surnuméraires ou indésirables dans la capitale. C'est encore largement le cas pour les simples conseillers municipaux ou régionaux. Au niveau des maires et des conseillers de région, les intéressés y voient souvent le point de chute opportun quand ils quitteront les ors de la République.

Car pour le reste la tâche n'est pas vraiment lourde compte tenu du fait que les moyens délégués aux communes et aux régions sont très limités. L'État ivoirien continue à se crisper sur le pré-carré de ses prérogatives et de ses budgets. S'il est vrai que la loi du 7 juillet 2003 transfère, de manière indistincte aux communes et aux départements (aujourd'hui régions), pas moins de seize compétences recouvrant la quasi-totalité des fonctions ministérielles, les activités réellement constatées sont très limitées. Les mairies assurent l'état civil, délivrent les autorisations d'occupation de l'espace public, entretiennent les marchés, et accompagnent parfois les activités socio-éducatives ou culturelles locales. En contrepartie, elles collectent des patentes et des taxes sur les marchés et dans les gares, ce qui constitue un enjeu financier non négligeable. Les régions 
pourront être amenées à réhabiliter des équipements scolaires, à équiper des centres de santé, à installer ou réparer des pompes, à condition d'avoir reçu les crédits délégués.

À l'expérience, on constate que les collectivités territoriales reçoivent rarement de l'État plus de $60 \%$ de ce qui a été prévu, voire promis, et que près de la moitié de cette dotation passe en crédits de fonctionnement. On note toutefois que les principales tâches administratives sont assurées par des fonctionnaires d'État détachés, car il n'existe pas de fonction publique territoriale. Il est d'ailleurs peu probable que les Institutions de Bretton Woods en acceptent le principe.

En Côte d'Ivoire comme ailleurs en Afrique subsaharienne, on est donc loin de la dynamique de développement qui devait accompagner les processus de décentralisation, même si de nombreuses opérations internationales relevant de la coopération décentralisée permettent un affichage souvent flatteur. Il n'est d'ailleurs pas exclu que la longue crise ivoirienne ait fait prendre du retard au pays, par rapport à certains programmes développés ailleurs en Afrique de l'Ouest.

\section{Conclusion}

On serait tenté de se demander si ces élections locales avaient du sens, à part la nécessité pour le pouvoir en place de respecter les échéances et les règles de la démocratie. En l'occurrence, ces consultations n'ont pas été inclusives puisque l'un des trois grands partis les a boycottées. Elles n'ont pas donné lieu à un renouvellement des élites politiques, puisque ce sont les vieux cadres qui ont monopolisé les meilleurs postes. Et elles ne déboucheront guère sur une dynamisation du développement local ou régional faute d'avoir donné à la loi de 2003 les moyens d'une réelle application.

Par ailleurs, elles n'ont pas vraiment réconcilié la Côte d'Ivoire avec la démocratie. En effet, les autorités n'ont toujours pas tenu compte des électeurs écartés des listes électorales. Puis il y a eu le blocage du FPI sur son refus de reconnaître sa défaite de 2010, et son recours - anachronique et pénalisant pour lui-même - au boycott. Mais, surtout, on a vu apparaitre une étrange sorte de quatrième grand parti : celui des «indépendants ». Par leur nombre et par leur identité réelle de dissidents, la plupart des candidats « indépendants » ont démontré que les vieux partis n'avaient guère su évoluer depuis le temps où Félix Houphouët-Boigny bâtissait lui-même les listes de ceux qui allaient être élus. Des notions aussi évidentes que le projet, l'implantation, la jeunesse ou le dynamisme passent encore après les petits calculs politiciens, les renvois d'ascenseur et les jeux de barbichette.

Il subsiste donc quelques malentendus sérieux à propos de la démocratie représentative. À quelques mois de la prochaine élection présidentielle, cela pourrait être inquiétant. Heureusement, on a aussi pu observer que le parti au pouvoir n'avait ni triché ni verrouillé, puisqu'il a admis des défaites peu glorieuses, dont celle de son secrétaire général.

Et la perspective du lancement d'un grand recensement général de la population laisse espérer que tous les Ivoiriens en âge de voter seront sollicités lors du prochain scrutin. À condition, naturellement, que l'actuelle opposition ne réactive pas le poison de l'ivoirité et accepte que des centaines de milliers de personnes soient intégrées dans le corps électoral. Seuls les Ivoiriens doivent avoir le droit de voter, mais tous doivent pouvoir le faire, sinon le pays replongera dans la situation de septembre 2002. 


\section{BIBLIOGRAPHIE}

Ballo Z., 2008. Décentralisation en Côte d'Ivoire : un bilan du fonctionnement des conseils généraux et des districts. CAPE-CIRES, $35 \mathrm{p}$.

Bouquet C., 2007. Le mauvais usage de la démocratie en Côte d'Ivoire. L'Espace politique, $\mathrm{n}^{\circ} 3$, décembre 2007, p. 90-106, http://www.espacepolitique.org/documents/pdf/

EP03_2007_F_bouquet_civ.pdf

Bouquet C., 2011. Côte d'Ivoire : le désespoir de Kourouma. A. Colin, 332 p.

Bouquet C., Kassi-Djodjo I., 2012. Les élections législatives en Côte d'Ivoire marquent-elles la sortie de crise ? EchoGéo [En ligne], Sur le Vif, mis en ligne le 26 mars 2012 http:// echogeo.revues.org/13017 ; DOI : 10.4000/echogeo.13017

Magrin G., 2006. La décentralisation réglera les problèmes de l'État en Afrique. L'Afrique des idées reçues, Belin 400 p., p. 383-389.

Nach Mback C., 2003. Démocratisation et décentralisation : genèse et dynamiques comparées des processus de décentralisation en Afrique subsaharienne. Karthala, $528 \mathrm{p}$.

Yapi Dihaou A., 1990. L'État et les municipalités en Côte d'Ivoire : un jeu de cache-cache ? Politique Africaine, $\mathrm{n}^{\circ} 40$, p. 51-59.

\section{NOTES}

1. Le RGPH 2013 (Recensement Général de la Population et de l'Habitat) a été officiellement annoncé en juin 2013. Il devait avoir lieu du 4 novembre au 3 décembre 2013, et produire ses premiers résultats «entre 2013 et 2014 » selon Mabri Toikeusse, ministre du Plan et du Développement (Le Démocrate, 01/07/2013). Pour des raisons matérielles, il a été reporté à mars 2014.

2. Une nouvelle loi sur la nationalité a été adoptée par le Parlement le 20 août 2013, mais nul n'est en mesure d'en chiffrer les bénéficiaires ( On verra bien ce que ça donnera » a déclaré à RFI le député indépendant Adolphe Konan Saraka, le 21/08/2013)...

3. $\mathrm{FPI}=$ Front populaire ivoirien ( socialiste $)$; RDR = Rassemblement des Républicains (« libéral », dissidence ancienne - 1994 - du PDCI) ; PDCI = Parti démocratique de Côte d'Ivoire (ex-parti unique de Félix Houphouët-Boigny).

4. La Commission électorale indépendante avait conservé la composition décidée à Pretoria en 2005 sur la base des « équilibres » de Marcoussis. Les ex-rebelles, pourtant officiellement dissous, disposaient donc d'assez nombreux sièges. 


\section{RÉSUMÉS}

Installé dans la douleur en mai 2011, le nouveau pouvoir ivoirien est parvenu à tenir le calendrier électoral de la mandature 2010-2015. Après les législatives de décembre 2011, les élections locales (municipales et régionales) ont eu lieu dans le calme en avril 2013. Mais le processus démocratique a conservé les mêmes faiblesses : découpage modifié, liste électorale incomplète, commission électorale indépendante non renouvelée. En outre, le FPI (parti de l'ancien chef de l'État) a de nouveau boycotté les consultations, et de nombreuses dissidences ont brouillé les scores des deux autres grands partis (RDR et PDCI), donnant aux «indépendants » un poids électoral difficile à déchiffrer. Heureusement, les enjeux étaient minimes car la décentralisation est encore très largement virtuelle.

Installed in difficulty in May 2011, the new Ivorian regime managed to keep the electoral calendar of the 2010-2015 mandature. After the legislatives of December 2011, local elections (municipal and regional) took place in a calm in April 2013. Therefore, the democratic process has lugged the same weaknesses (incomplete list of electors, unrenowned independent electoral commission). In addition, the FIP (the party of former Head of State) has once more boycotted the consultations and many divisions inside the others two major parties (RDR and PDCI) have blurred their scores and gave to "independents" an electoral weight difficult to decipher. Fortunately, the issues were minimal due the fact that the decentralization is still largely virtual.

\section{INDEX}

Mots-clés : Afrique, démocratie, Côte d'Ivoire, élection locale

Keywords : Africa, democracy, Côte d'Ivoire, local election

\section{AUTEURS}

\section{CHRISTIAN BOUQUET}

Christian Bouquet, christian.bouquet@u-bordeaux3.fr, est Professeur émérite à l'université Michel de Montaigne (Bordeaux 3), membre de l'UMR LAM (Les Afriques dans le Monde).

\section{IRÈNE KASSI-DJODJO}

Irène Kassi-Djojo, irenekassi@yahoo.fr, est Maître-assistante à l'université Félix HouphouëtBoigny (Abidjan), Institut de Géographie tropicale (IGT). 